Research Article

\title{
New Traveling Wave Solutions to the Vakhnenko-Parkes Equation
}

\author{
XiaoHua Liu and Caixia He \\ The School of Sciences, Guizhou Minzu University, Guiyang, Guizhou 550025, China \\ Correspondence should be addressed to XiaoHua Liu; lxhjkkl@yeah.net
}

Received 12 June 2013; Accepted 29 July 2013

Academic Editors: G. Cleaver, J. Garecki, and D. Singleton

Copyright (C) 2013 X. Liu and C. He. This is an open access article distributed under the Creative Commons Attribution License, which permits unrestricted use, distribution, and reproduction in any medium, provided the original work is properly cited.

We apply the improved $\left(G^{\prime} / G\right)$ expansion method to the Vakhnenko-Parkes equation. As a result, many new and more general exact solutions have been obtained for the equation. Comparing our solutions with those gained by other authors indicates that the improved $\left(G^{\prime} / G\right)$ expansion is more effective in solving the general solutions to differential equations.

\section{Introduction}

Many phenomena in physics and other fields such as biology chemistry and mechanics. are described by nonlinear partial differential equations (NLPDEs). The investigation of traveling wave solutions to nonlinear partial differential equations (NLPDEs) plays an important role in the study of nonlinear physical phenomena. In the past several decades, both mathematicians and physicists have made significant progress in this direction.

Many effective methods [1-10] have been presented such as exp-function method [1], Hirota's method [2], variational iteration method [3], the homogeneous balance method [4], Backlund and Darboux transformation method [5], the sine-cosine function method [6], the Jacobi elliptic function method [7], and auxiliary equation method [8-10].

Recently, an interesting and important discovery has been made by Vakhnenko and Parkes [11], who have demonstrated that the reduced Ostrovsky equation [12]

$$
\left(u_{t}+c_{0} u_{x}+\alpha u u_{x}\right)_{x}=\gamma u
$$

can be transformed to the new integrable equation

$$
u u_{x x t}-u_{x} u_{x t}+u^{2} u_{t}=0
$$

In the literature, the traveling wave solutions of the Vakhnenko-Parkes equation (2) are investigated by the improved tanh function method introduced in $[13,14]$, auxiliary equation method [10], and $G^{\prime} / G$-expansion method [15].
The present paper is motivated by the desire to improve the work made in $[4,10,13-15]$ by proposing a new improved $G^{\prime} / G$-expansion method to construct more general exact solutions of nonlinear partial differential equations (NLPDES). For illustration, we restrict our attention to the study of the Vakhnenko-Parkes equation (2) and successfully construct many new and more general exact solutions.

The rest of this paper is organized as follows: we give the description of the improved $G^{\prime} / G$-expansion method in Section 2. In Section 3, we apply this method to (2). In Section 4 , some conclusions are given.

\section{Description of the Improved $G^{\prime} / G$-Expansion Method}

To make this paper entire, in here we enumerate the same method reported in [16]. Suppose that we have a NLPDE for $u(x, t)$ in the form

$$
P\left(u, u_{x}, u_{t}, u_{x t}, u_{x x}, \ldots\right)=0
$$

where $P$ is a polynomial in its arguments, which includes nonlinear terms and the highest order derivatives. The transformation $u(x, t)=u(\xi), \xi=x-\omega t$ reduces (3) to the ordinary differential equation (ODE)

$$
H\left(u, u_{\xi}, u_{\xi \xi}, u_{\xi \xi \xi}, \ldots\right)=0
$$


By virtue of the extended tanh-function method, we assume that the solution of (4) is in the following form

$$
u(\xi)=\sum_{i=0}^{m} a_{i} F^{i}(\xi)
$$

in which $a_{i}(i=0,1,2, \ldots, m)$ are all real constants to be determined and the balancing number $m$ is a positive integer which can be determined by balancing the highest derivative terms with the highest power nonlinear terms in (4), and $F(\xi)$ is

$$
F(\xi)=\frac{G^{\prime}(\xi)}{G(\xi)}
$$

where $G(\xi)$ expresses the solution of the following auxiliary ordinary differential equation:

$$
G G^{\prime \prime}=A G^{2}+B G G^{\prime}+C\left(G^{\prime}\right)^{2}
$$

where the prime denotes derivative with respect to $\xi . A, B$, and $C$ are real parameters.

Using the general solutions of (7) and with the help of Maple, we have the following.

Case 1. For $B \neq 0$ and $\Delta=B^{2}+4 A-4 A C \geq 0$,

$$
F(\xi)=\frac{B}{2(1-C)}+\frac{B \sqrt{\Delta}}{2(1-C)} \frac{c_{1} e^{(\sqrt{\Delta} / 2) \xi}+c_{2} e^{-(\sqrt{\Delta} / 2) \xi}}{c_{1} e^{(\sqrt{\Delta} / 2) \xi}-c_{2} e^{-(\sqrt{\Delta} / 2) \xi}} .
$$

Case 2. For $B \neq 0$ and $\Delta=B^{2}+4 A-4 A C<0$,

$$
\begin{aligned}
F(\xi)= & \frac{B}{2(1-C)} \\
& +\frac{B \sqrt{-\Delta}}{2(1-C)} \frac{i c_{1} \cos (\sqrt{-\Delta} / 2) \xi-c_{2} \sin (\sqrt{-\Delta} / 2) \xi}{i c_{1} \sin (\sqrt{-\Delta} / 2) \xi+c_{2} \cos (\sqrt{-\Delta} / 2) \xi} .
\end{aligned}
$$

Case 3. For $B=0$ and $A(C-1) \geq 0$,

$$
\begin{aligned}
F(\xi)= & \frac{\sqrt{A(C-1)}}{(1-C)} \\
& \times \frac{c_{1} \cos (\sqrt{A(C-1)} \xi)+c_{2} \sin (\sqrt{A(C-1)} \xi)}{c_{1} \sin (\sqrt{A(C-1)} \xi)-c_{2} \cos (\sqrt{A(C-1)} \xi)} .
\end{aligned}
$$

Case 4. For $B=0$ and $A(C-1)<0$,

$$
\begin{aligned}
F(\xi)= & \frac{\sqrt{-A(C-1)}}{(1-C)} \\
& \times \frac{i c_{1} \cosh (\sqrt{-A(C-1)} \xi)-c_{2} \sinh (\sqrt{-A(C-1)} \xi)}{i c_{1} \sinh (\sqrt{-A(C-1)} \xi)-c_{2} \cosh (\sqrt{-A(C-1)} \xi)} .
\end{aligned}
$$

Herein, $A, B, C$ and $c_{1}, c_{2}$ are real parameters.

\section{Traveling Wave Solutions for the Vakhnenko-Parkes Equation}

We now consider the improved $G^{\prime} / G$-expansion method to obtain the traveling wave solutions of (2); substituting

$$
u=u(\xi)=u(x-w t)
$$

into (2), integrating once with respect to $\xi$, and setting the integration constant equal to zero yield

$$
3 u u^{\prime \prime}-3 u^{\prime 2}+u^{3}=0
$$

Balancing $u^{3}$ with $u u^{\prime \prime}$ gives the leading order $m=2$, so we take the ansatz

$$
u(\xi)=a_{0}+a_{1} F(\xi)+a_{2} F^{2}(\xi)
$$

where $a_{0}, a_{1}$, and $a_{2}$ are constant and need to be determined ans $F(\xi)$ express the solution of (6). Substituting (14) and (7) along with (6) into (13) and using Maple yield a system of equations of $F^{i}$; setting the coefficients of $F^{i}(i=0,1,2, \ldots 6)$ in the obtained system of equations to zero, we can deduce the following set of algebraic polynomials with respect to unknown $a_{0}, a_{1}$, and $a_{2}$; namely,

$$
\begin{gathered}
6 a_{2}^{2}+6 a_{2}^{2} C^{2}-12 a_{2}^{2} C+a_{2}^{3}=0 \\
6 a_{2}^{2} B C-6 a_{2}^{2} B+3 a_{2}^{2} a_{1}+12 a_{1} a_{2} C^{2}-24 a_{1} a_{2} C+12 a_{1} a_{2}=0 \\
-6 a_{1}^{2} C+15 a_{1} a_{2} C B-15 a_{1} a_{2} B+18 a_{0} a_{2} C^{2}-36 a_{0} a_{2} C \\
+3 a_{0} a_{2}^{2}+3 a_{1}^{2}+3 a_{1}^{2} a_{2}+18 a_{0} a_{2}+3 a_{2}^{2} C^{2}=0 \\
-12 a_{0} a_{1} C+6 a_{1} a_{2} A C-3 a_{1}^{2} B+3 a_{1}^{2} B C-6 a_{2}^{2} A B \\
+6 a_{0} a_{1} a_{2}+3 a_{1} a_{2} B^{2}+6 a_{0} a_{1}+30 a_{0} a_{2} B C \\
-6 a_{1} a_{2} A-30 a_{0} a_{2} B+a_{1}^{3}+6 a_{0} a_{1} C^{2}=0 \\
-6 a_{2}^{2} A^{2}-9 a_{0} a_{1} B-3 a_{1} a_{2} A B+3 a_{0} a_{1}^{2}+3 a_{0}^{2} a_{2} \\
+24 a_{0} a_{2} A C+9 a_{0} a_{1} B C-24 a_{0} a_{2} A+12 a_{0} a_{2} B^{2}=0 \\
3 a_{0} a_{1} B^{2}+6 a_{0} a_{1} A C-3 a_{1}^{2} A B-6 a_{0} a_{1} A+3 a_{0}^{2} a_{1} \\
-6 a_{1} a_{2} A^{2}+18 a_{0} a_{2} A B=0 \\
6 a_{0} a_{2} A^{2}+3 a_{0} a_{1} A B-3 a_{1}^{2} A^{2}+a_{0}^{3}=0
\end{gathered}
$$

Solving the set of algebraic equations by the use of Maple, we get the following results:

$$
\begin{gathered}
a_{0}=-6 A C+6 A ; \quad a_{1}=-6 B(C-1) ; \\
a_{2}=-6(C-1)^{2} .
\end{gathered}
$$

Substituting (16) and (12) along with $F(\xi)$ in (8), (9), (10), and (11) into (14), we obtain the following exponential 
function solutions, hyperbolic function solutions, and triangular function solutions of (2). These solutions are as follows:

(1) when we choose $B \neq 0$ and $\Delta=B^{2}+4 A-4 A C \geq 0$, then the exponential function solutions can be found as

$$
\begin{aligned}
u(x, t) & \\
= & -6 A C+6 A+\frac{3}{2} B^{2} \\
& -\frac{3}{2} \Delta B^{2}\left[\frac{c_{1} e^{(\sqrt{\Delta} / 2)(x-\omega t)}+c_{2} e^{-(\sqrt{\Delta} / 2)(x-\omega t)}}{c_{1} e^{(\sqrt{\Delta} / 2)(x-\omega t)}-c_{2} e^{-(\sqrt{\Delta} / 2)(x-\omega t)}}\right]^{2} ;
\end{aligned}
$$

(2) when we choose $B \neq 0$ and $\Delta=B^{2}+4 A-4 A C<0$, the triangular function solutions will be

$$
\begin{aligned}
& u(x, t) \\
& =-6 A C+6 A+\frac{3}{2} B^{2}+\frac{3}{2} \Delta B^{2} \\
& \quad \times\left[\frac{i c_{1} \cos (\sqrt{-\Delta} / 2)(x-\omega t)-c_{2} \sin (\sqrt{-\Delta} / 2)(x-\omega t)}{i c_{1} \sin (\sqrt{-\Delta} / 2)(x-\omega t)+c_{2} \cos (\sqrt{-\Delta} / 2)(x-\omega t)}\right]^{2} ;
\end{aligned}
$$

(3) if we choose $B=0$ and $A(C-1) \geq 0$, then the triangular function solutions are

$$
\begin{aligned}
& u(x, t) \\
& =-6 A C+6 A-6 A(C-1) \\
& \quad \times\left[\frac{c_{1} \cos (\sqrt{A(C-1)}(x-\omega t))+c_{2} \sin (\sqrt{A(C-1)}(x-\omega t))}{c_{1} \sin (\sqrt{A(C-1)}(x-\omega t))-c_{2} \cos (\sqrt{A(C-1)}(x-\omega t))}\right]^{2} ;
\end{aligned}
$$

(4) again, when we choose $B=0$ and $A(C-1)<0$, then hyperbolic function solutions are

$$
\begin{aligned}
& u(x, t) \\
& =-6 A C+6 A+6 A(C-1) \\
& \quad \times\left[\frac{i c_{1} \cosh (\sqrt{-A(C-1)}(x-\omega t))-c_{2} \sinh (\sqrt{-A(C-1)}(x-\omega t))}{i c_{1} \sinh (\sqrt{-A(C-1)}(x-\omega t))-c_{2} \cosh (\sqrt{-A(C-1)}(x-\omega t))}\right]^{2},
\end{aligned}
$$

where $A, B, C$ and $c_{1}, c_{2}$ are real parameters.

\section{Conclusion and Discussion}

In this work, we have presented an improved $G^{\prime} / G$-expansion method and applied it to obtain new traveling wave solutions of the Vakhnenko-Parkes equation. In contrast to other $G^{\prime} / G$-expansion methods, some benefits are available for this method.

First, all the nonlinear PDEs which can be solved by other $G^{\prime} / G$-expansion methods can be solved easily by this method. We have successfully obtained many new exact traveling wave solutions. To our knowledge, these solutions have not been reported in the former literature.
Second, if we used the special value of parameters $c_{1}, c_{2}$ and $A, B, C$, we can obtain some traveling wave solutions which have been found by Yaşar [13], such as the following.

If we suppose that $B^{2}=1, A-A C=3 / 4$, the exponential function solutions (17) at $c_{1}=c_{2}$ can be rewritten as follows:

$$
u(x, t)=6-6 \operatorname{coth}^{2}(x-\omega t),
$$

while at $c_{1}=-c_{2}$, they yield

$$
u(x, t)=6-6 \tanh ^{2}(x-\omega t) .
$$

Similarly, when we choose $A-A C=-1$, it is easy to see that the trigonometric function solutions (19) at $c_{1} \neq 0, c_{2}=0$ can be rewritten as follows:

$$
u(x, t)=-6-6 \cot ^{2}(x-\omega t),
$$

while at $c_{1}=0, c_{2} \neq 0$, one can obtain

$$
u(x, t)=-6-6 \tan ^{2}(x-\omega t) .
$$

In [3], the authors yield (21), (22), (23), and (24) by using tanh function method.

On the other hand, in [10], Kangalgil and Ayaz have derived a traveling wave solution

$$
u(x, t)=\frac{3}{2} a\left[2(1+\varepsilon \operatorname{coth} \eta)-(1+\varepsilon \operatorname{coth} \eta)^{2}\right]
$$

by the auxiliary equation method, where $\eta=\sqrt{a} / 2, a>0$.

Choosing $B^{2}=1, \Delta=a$, and $c_{1}=c_{2}$ in (17) and after some simplifications, we obtain (25) at $\varepsilon=1$.

\section{Acknowledgments}

This project is supported by the Technical Innovation Talents Support Plan of Guizhou Education Department (KY[2012]092) and Fund of Guizhou Science and Technology Department ([2013]2138).

\section{References}

[1] W.-X. Ma, T. Huang, and Y. Zhang, "A multiple exp-function method for nonlinear differential equations and its application," Physica Scripta, vol. 82, no. 6, Article ID 065003, 2010.

[2] W.-X. Ma and E. Fan, "Linear superposition principle applying to Hirota bilinear equations," Computers \& Mathematics with Applications, vol. 61, no. 4, pp. 950-959, 2011.

[3] J.-H. He, "Variational iteration method-some recent results and new interpretations," Journal of Computational and Applied Mathematics, vol. 207, no. 1, pp. 3-17, 2007.

[4] M. Wang, Y. Zhou, and Z. Li, "Application of a homogeneous balance method to exact solutions of nonlinear equations in mathematical physics," Physics Letters A, vol. 216, no. 1-5, pp. 6775, 1996.

[5] G. L. Lamb, Jr., "Bäcklund transformations for certain nonlinear evolution equations," Journal of Mathematical Physics, vol. 15, pp. 2157-2165, 1974.

[6] A.-M. Wazwaz, "New travelling wave solutions of different physical structures to generalized BBM equation," Physics Letters $A$, vol. 355, no. 4-5, pp. 358-362, 2006. 
[7] E. V. Krishnan, "On the Itô-type coupled nonlinear wave equation," Journal of the Physical Society of Japan, vol. 55, no. 11, pp. 3753-3755, 1986.

[8] S. Zhang, "A generalized new auxiliary equation method and its application to the $(2+1)$-dimensional breaking soliton equations," Applied Mathematics and Computation, vol. 190, no. 1, pp. 510-516, 2007.

[9] E. Yomba, "A generalized auxiliary equation method and its application to nonlinear Klein-Gordon and generalized nonlinear Camassa-Holm equations," Physics Letters A, vol. 372, no. 7, pp. 1048-1060, 2008.

[10] F. Kangalgil and F. Ayaz, "New exact travelling wave solutions for the Ostrovsky equation," Physics Letters A, vol. 372, no. 11, pp. 1831-1835, 2008.

[11] V. O. Vakhnenko and E. J. Parkes, “The two loop soliton solution of the Vakhnenko equation," Nonlinearity, vol. 11, no. 6, pp. 1457-1464, 1998.

[12] L. A. Ostrovsky, "Nonlinear internal waves in a rotating ocean," Oceanology, vol. 18, pp. 119-125, 1978.

[13] E. Yaşar, "New travelling wave solutions to the Ostrovsky equation," Applied Mathematics and Computation, vol. 216, no. 11, pp. 3191-3194, 2010.

[14] E. Yusufoğlu and A. Bekir, "A travelling wave solution to the Ostrovsky equation," Applied Mathematics and Computation, vol. 186, no. 1, pp. 256-260, 2007.

[15] R. Abazari, "Application of $\left(G^{\prime} / G\right)$-expansion method to travelling wave solutions of three nonlinear evolution equation," Computers \& Fluids, vol. 39, no. 10, pp. 1957-1963, 2010.

[16] X. Liu, W. Zhang, and Z. Li, "Application of improved $\left(G^{\prime} / G\right)$ expansion method to traveling wave solutions of two nonlinear evolution equations," Advances in Applied Mathematics and Mechanics, vol. 4, no. 1, pp. 122-130, 2012. 


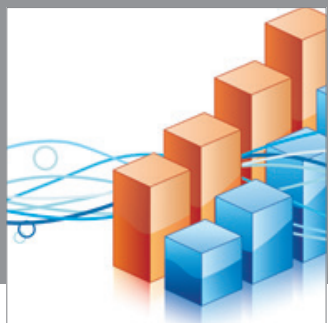

Advances in

Operations Research

mansans

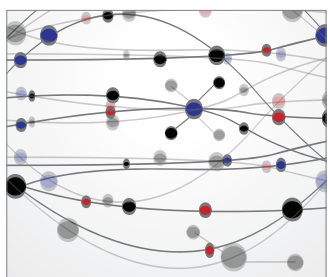

The Scientific World Journal
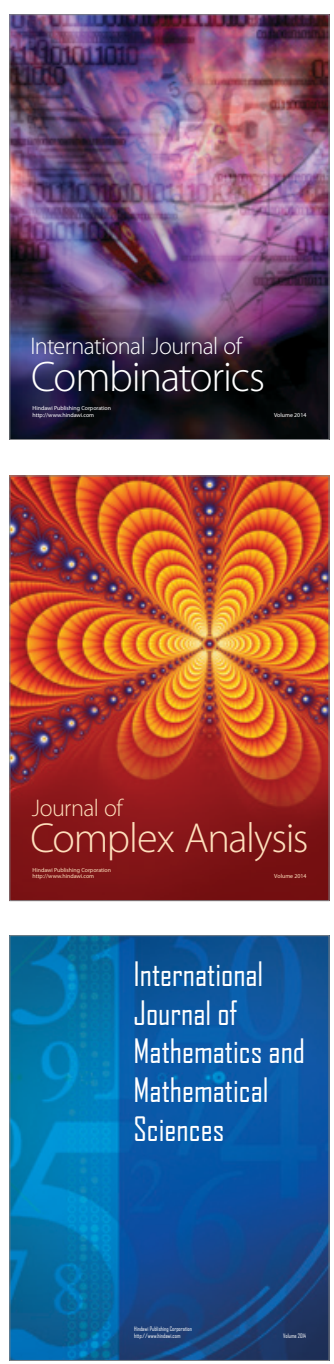
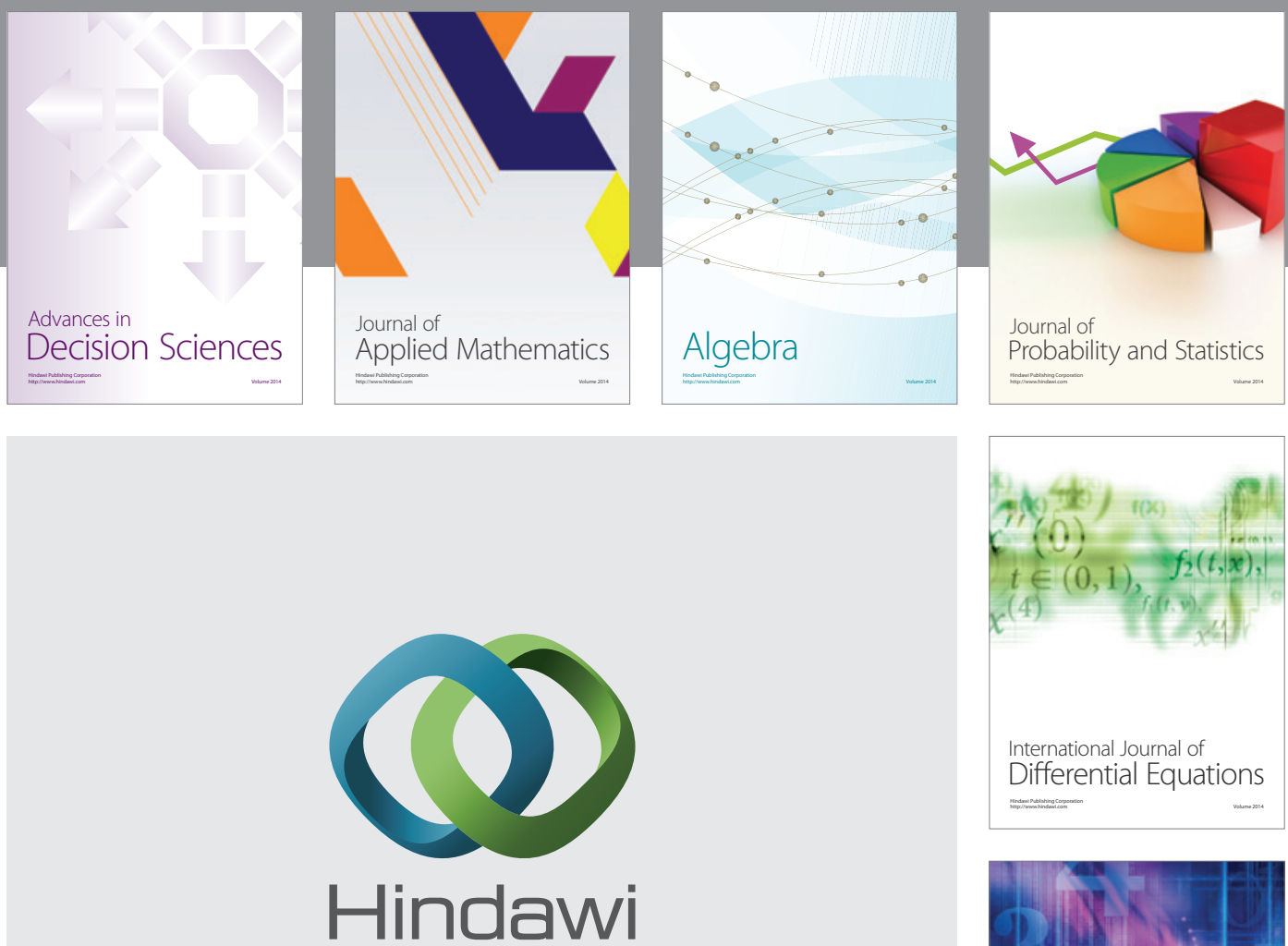

Submit your manuscripts at http://www.hindawi.com
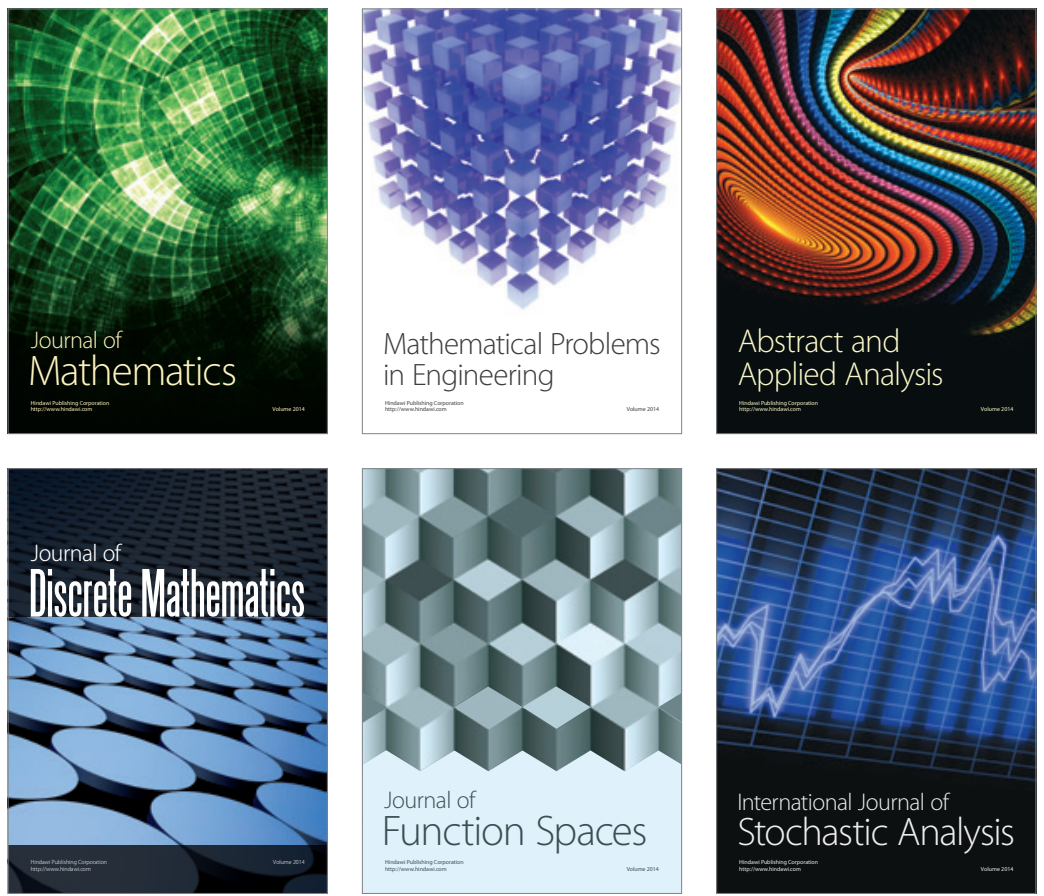

Journal of

Function Spaces

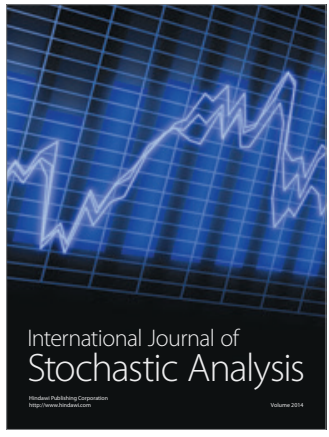

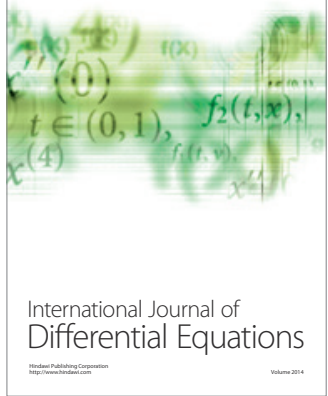
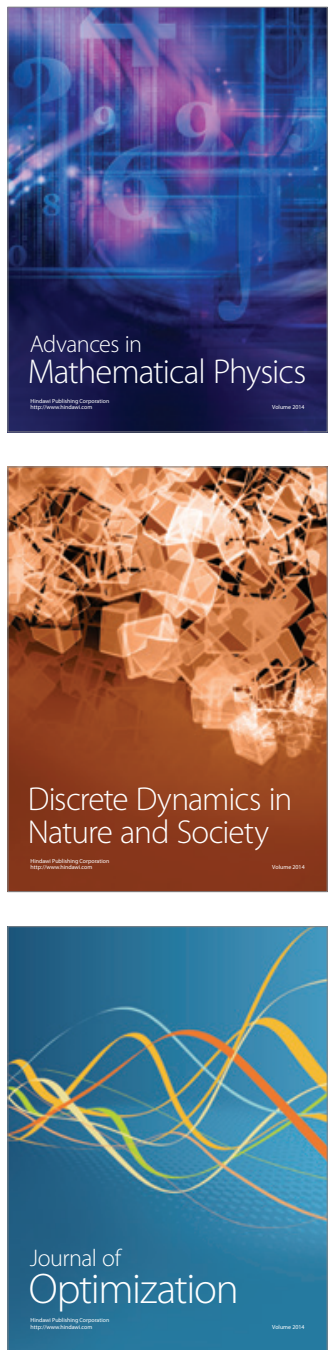